\title{
BIOANALYSIS METHOD DEVELOPMENT AND VALIDATION OF SMALL MOLECULES IN PHARMACEUTICAL INDUSTRY: A BIOANALYST REVIEW POINT
}

\author{
SATHESHKUMAR $\mathbf{S}^{1 *}$, MURUGANANTHAM V ${ }^{1}$, KANAGA SABAPATHI $\mathbf{S}^{2}$, CHITHRA $^{3}$
}

${ }^{1}$ Department of Pharmaceutics, College of Pharmacy, Vinayaka Mission's College of Pharmacy, Salem, Tamil Nadu, India. ${ }^{2}$ Department of Bioanalytical, Drug Metabolism and Pharmacokinetics, Anthem Biosciences Private Limited, Bengaluru, Karnataka, India. ${ }^{3} \mathrm{Quality}$ Control, Bioanalytical Research Laboratory, Syngene International Limited, Bengaluru, Karnataka, India. Email: sat2794@gmail.com

Received: 16 December 2020, Revised and Accepted: 22 January 2021

ABSTRACT

The focus of bioanalysis employed for the quantitative determination of an active analyte(s) and their metabolite(s) in the biological matrix such as plasma, serum, blood, cerebrospinal fluid, and tissues. The extraction of analyte and metabolite in the biological fluids is carried out using different separation methods such as protein precipitation, liquid-liquid extraction, and solid phase extraction. Bioanalytical method development and validation in the pharmaceutical industry are to provide an assessment and interpretation of pharmacokinetics, pharmacodynamics, toxicokinetics, bioavailability/bioequivalence, and therapeutic drug monitoring relationships. This review paper aims to provide a simple and accurate scientific background to improve the quality for development and validation of a bioanalytical method for small molecules with industrial technique as per regulatory agency requirements (United States Food and Drug Administration, EMEA, International Council for Harmonisation and ANVISA).

Keywords: Matrix factor, Recovery, Stability, Quality control, Liquid chromatography-mass spectrometry.

(c) 2021 The Authors. Published by Innovare Academic Sciences Pvt Ltd. This is an open access article under the CC BY license (http://creativecommons.org/ licenses/by/4.0/) DOI: http://dx.doi.org/10.22159/ajpcr.2021v14i3.40528. Journal homepage: https://innovareacademics.in/journals/index.php/ajpcr

\section{INTRODUCTION}

The bioanalysis is a subdiscipline of analytical chemistry for the quantitative measurement of xenobiotics and biotics in biological systems and it plays a very important role in drug development, the conduct of clinical trials, and in therapeutic drug monitoring (TDM). Various analytical procedures have been developed to execute bioanalysis, which includes gas chromatography (GS)-flame ionization detection, immunoassays, GS-mass spectrometry (MS), highperformance liquid chromatography (HPLC)-ultraviolet, HPLC-MS (HPLC-MS or LC-MS), and HPLC tandem MS (HPLC-MS/MS or LC-MS/ MS) $[1,2]$.

New drug development is a lengthy and costly procedure. It normally lasts 15-17 years and roughly 1 out of 5000 compounds may come out as marketable drug. The most frequently used techniques in bioanalytical studies are HPLC tandem MS (HPLC-MS/MS or LC-MS/ MS). Roles of LC-MS bioanalysis in drug discovery, development, and TDM is given in Fig. 1 [3-5].

Bio-analytical validation confirms the high-quality data for regulatory submission as well as for the drug discovery and development [6]. The first guideline released by the United States Food and Drug Administration (USFDA) for the bioanalytical method validation in May 2001 which were further amended in May 2012 and presently final version released May 2018 [7-9]. National Health Surveillance Agency (ANVISA), Brazil released its first bioanalytical guidelines in May 2003 in combination with analytical validation guidelines, which were further amended in May 2012 [10,11]. European Medicines Agency (EMA, European authority) issued its guidelines which became effective since February 2012 [12]. Ministry of Health, Labour and Welfare MHLW, Japan issued draft guideline for bioanalytical methods in 2013 [13]. International Council for Harmonisation (ICH) issued its draft guidelines which became since Feb 2019 [14].

\section{Reference standard}

Bioanalysis of drugs and their metabolites are carried out using samples spiked with calibration (reference) standards and Quality
Control samples. The reference standard purity of spiked samples can affect study results. Hence, a certified analytical reference standard of known identity and purity should be used to prepare solutions of labeled concentrations. If at all possible, the reference standard should be identical to the analyte, not possible; an established chemical form (free acid, base, and salt) of known purity can be used. Three types of reference standards are used:

i. Certified reference standards (e.g., USP [U.S. Pharmacopoeia] standards)

ii. Commercially supplied reference standards obtained from a reputable commercial source

iii. Custom synthesized materials with documented purity by an analytical laboratory.

The analyte source and batch/lot number, expiry date, certificates of analyses available, and internally or externally created confirmation of identity and purity of each reference standard. If the reference standard expires, should not make stock solutions for analysis with this batch/ lot of reference standard unless the reference standard's purity is reestablished $[9,15]$.

\section{METHOD DEVELOPMENT}

A bioanalytical method is a set of procedures involved in the collection, processing, storage, and analysis of biological matrices (e.g.: Plasma, urine, cerebrospinal fluid, etc.) for a chemical compound.

\section{Goals for sample preparation [16]}

Remove unwanted interferences (proteins, salts, metabolites, endogenous substances)

- Solubilize analytes [16]

- Remove particulates that may block chromatographic tubing

- Concentrate analytes to achieve sensitivity gains

- Dilute analyte concentrations or solvent strength when outside the range of detection

- Modify pH to promote or counteract ionization

- Exchange the solvent in which the analyte resides (e.g., aqueous to a nonpolar solvent) 


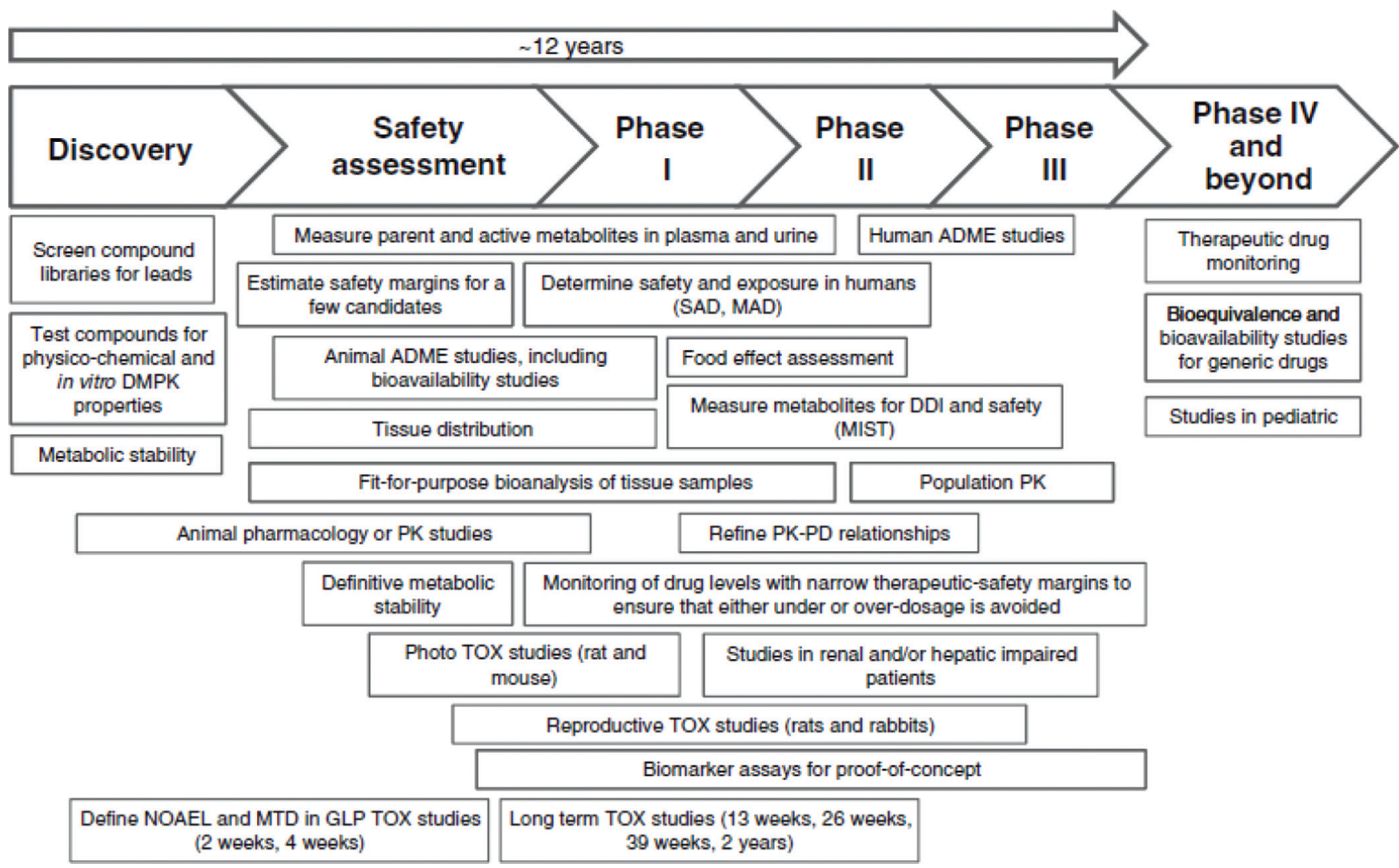

Fig. 1: Roles of liquid chromatography- mass spectrometry bioanalysis in pharmaceutical industry

- Derivatize or complex with a chemical species to improve detection sensitivity

- Remove an unwanted metabolic functional group (e.g., glucuronide conjugate)

- Remove proteins and lipids that can cause ion suppression

- Hydrolyze large proteins for peptide analysis

- Disrupt analyte binding to protein or another component within the sample matrix.

\section{Protein precipitation (PPT)}

PPT is a common choice for sample preparation method because it involves simply a one-step addition of solvent to the sample and is considered a universal procedure (requiring no method development). The speed of this technique is very attractive to analysts, as well as its low cost and time. The high resolving power of LC-MS/MS analytical methods generally overcomes the non-selective cleanup procedure. Typical sample matrices that are used with PPT techniques are plasma, serum, whole blood, tissue homogenates, and in vitro incubation mixtures [88-90].

A general approach to eliminate proteins from a biological sample is the addition of an organic solvent (methanol, ethanol, or acetonitrile). Typically, a volume of sample matrix (1 part) is diluted with a volume of the precipitating agent (2-4 parts), followed by vortex mixing and then centrifugation or filtration to isolate or remove the mass of precipitated protein. An aliquot of the supernatant or filtrate is injected for bioanalysis [16,17].

\section{Liquid-liquid extraction (LLE)}

LLE is a technique used to separate analytes from interferences in a sample matrix by the act of partitioning between two immiscible liquids under the optimal conditions, the analyte in solution will preferentially migrate into the organic solvent. At present, liquid extraction replaced with advanced and enhanced methods like liquidphase microextraction and supported membrane extraction, single drop liquid-phase microextraction [16-18].

\section{Solid phase extraction (SPE)}

The main goals of SPE are the elimination of interfering matrix components and selective concentration and isolation of the analytes.

\section{SPE protocols}

Typical SPE protocols for trace analysis involve following steps

1. Wetting and conditioning - to solvate and activate the sorbent to retain analytes of interest

2. Sample loading/retention - application of the sample to the cartridge (sorbent) where analytes of interest are retained

3. Wash/rinse-anylesser retained interferences are washed off the sorbent

4. Elution - removal of analytes of interest from sorbent into a collection tube using a relatively small volume of a suitable solvent (elution solvent).

To ensure optimum target analyte retention and, depending on the sorbent chemistry, it may be necessary to pretreat the sample $[18,19]$.

\section{METHOD VALIDATION}

Bioanalytical method validation is the process used to establish the qualitative and quantitative determination analyte and metabolites in the biological matrix.

\section{Types of method validation}

Full validation

Full method validation is mentioned by all the regulatory agency (USFDA, EMEA, and ANVISA) for the developing and implementing a new bioanalytical method for the analysis of new drug entity and or its metabolites(s) or biomarkers. Full validation of the revised assay needs to perform if quantification of metabolites is added to the existing assay or new analyte/metabolite(s) $[7,20]$.

\section{Partial validation}

Partial validations evaluate changes in the already validated bioanalytical methods. It can range from as little as one intra assay 
accuracy and precision (A\&P) determination to nearly full validation. Raw data on partial validations should be retained at the analytical site for inspection when requested. Typical bioanalytical method modifications or changes that fall into this category include, but are not limited to the following $[7,20]$,

- Bioanalytical method transfer between laboratories or analysts

- Modification in the analytical procedure

- Modification in anticoagulant in harvesting biological fluid (e.g., $\mathrm{K}_{2}$ EDTA to Heparin)

- Modification in a matrix within species (e.g., human urine to human plasma)

- Modification in the sample extraction procedure

- Modification in species within the matrix (e.g., mouse plasma to rat plasma)

- Modification in a significant concentration range

- Modification in instrument and software platforms

- Limited sample volume/Rare matrices.

Cross-validation

Cross method validation is a comparison of validation parameters when two or more bioanalytical methods are used to generate data within a similar study or crosswise different studies. Cross-validation needs to consider it if sample analysis within a single study is conducted at more than one site or more than one laboratory. In cases, cross-validation with shared matrix quality control samples and non-pooled subjects samples should be conducted at each site or laboratory to establish inter-laboratory reliability $[21,22]$.

\section{Validation parameters}

System suitability

The system suitability test of the chromatographic system was performed to confirm the adequate suitability and reproducibility of the system before each day of a validation run. A system suitability test was performed by injecting six consecutive injections using a standard aqueous mixture equivalent to middle concentration of the calibration standard curve of analyte and working concentration of ISTD [23].

\section{Acceptance criteria}

The $\%$ coefficient of variation (CV) for the peak area response ratio was found to be $\leq 5.0 \%$ and that for retention time was $\leq 2.0 \%$.

\section{Autosampler carry over test}

This denotes that a compound is getting carried over from one injection to the next. The chromatographic system should be tested to evaluate the possibility of carry over by injecting mobile phase followed by an extracted blank, extracted lower limit of quantification (LLOQ), extracted ULOQ and then once again extracted blank $[22,23]$.

\section{Acceptance criteria}

The response of interference at analyte RT should not be more than or equal to $20 \%$ of extracted LLOQ response for the drug and $5 \%$ of internal standard response at ISTD RT in a reinjected blank sample after extracted ULOQ sample.

\section{Selectivity/specificity}

Selectivity/specificity is the extent to which the method can determine a particular analyte of interest in the analyzed matrices without interference from endogenous components, internal standard, multiple analyte(s), co-administered drug, and concomitant medication drugs.

Process minimum of six individual blank matrix lots including one hemolyzed and one lipemic matrix from independent sources as per the method with respective lots LLOQ level. The impact of hemolyzed/ lipemic samples or samples from special populations can be included in the selectivity assessment. Evaluate the interference in a blank matrix by comparing the response at the retention time of analyte(s) and ISTD, against LLOQ spiked sample $[7,24,25]$.

\section{Acceptance criteria}

- Analyte response in STD Blank should not exceed $20 \%$ of respective LLOQ sample of matrix and LLOQ response should be $\pm 20 \%$ of nominal concentration

- The ISTD response in the STD blank should not exceed 5\% of the average ISTD responses of the accepted calibrators and QCs

- Evaluate the $80 \%$ of tested lots are free from interference at the retention time of the analyte and internal standard [41-44].

\section{Sensitivity}

This is the lowest possible amount of analyte that can be detected and distinguished from noise in the instrument with adequate A\&P. A minimum of six replicates of LLOQ samples shall be analyzed with a freshly prepared calibration curve for the sensitivity assessment. The LLOQ evaluation shall be done separately or as part of the precision and accuracy assessment for the calibration range $[7,26]$.

\section{Acceptance criteria}

The precision of LLOQ samples should be $\leq 20 \%$ and mean $\%$ bias of LLOQ samples should be within $\pm 20 \%$. The individual $\%$ bias of at least $67 \%$ (4 out of 6 ) of LLOQ samples should be within $\pm 20 \%$.

\section{Detection limit}

According to ICH, it is the lowest concentration of an analyte in a sample which can be detected but not necessarily quantified as an exact value. According to Conference Report II, it is the lowest concentration of an analyte in a sample that the bioanalytical procedure can reliably differentiate from background noise [27].

\section{Linearity/calibration curve}

The calibration curve also known as the standard curve is the relationship between the instrument response and the calibration standards within the intended quantitative concentration range. It constructed using calibration standards are used to back-calculate the concentrations of quality control samples and if applicable (subject) samples [34-40,45].

Calibration curve/linearity shall contain (standard) blank (no analyte, no ISD), zero calibrators/standard zero (blank plus ISTD(s)), and at least six non-zero calibrator levels covering the quantitation range, including LLOQ in every run and evaluate at least 3 calibration curves generated using spiked samples. The concentration-response relationship, as well as an appropriate weighting scheme and regression equation, should be established [28,29].

\section{Acceptance criteria}

- The accuracy (\% bias) of LLOQ/STD-01 from nominal concentration should be within $\pm 20 \%$ and for standards other than LLOQ; accuracy (\% bias) should be within $\pm 15 \%$

- At least $75 \%$ non-zero standards should meet the above criteria, including the LLOQ and the calibration standard at the highest concentration

- The goodness for fit value should be greater than or equal to 0.98

- Only data points that fail to meet the acceptance criteria shall be excluded from the study.

\section{A\&P}

The precision of the assay was measured by the percentage coefficient variation over the concentration range of quality control samples of analyte and metabolite during the validation and the accuracy of the assay was defined as the absolute value of the ratio of calculated mean concentration values of the quality control samples to their respective nominal values [90-94].

A\&P should be established with at least three independent A\&P runs, four QC levels per run (LLOQ, LQC, MQC, and HQC), and six replicates per QC level. With intra-inter run A\&P. A\&P experiments shall be performed using freshly prepared calibration standards and freshly prepared QCs [28-29]. 


$$
\begin{aligned}
& \text { Accuracy }(\% \text { Bias })=\frac{\text { Calculated Concentration }}{\text { Nominal Concentration }} \times 100 \\
& \text { Precision }(\% \mathrm{CV})=\frac{\text { Standard Deviation }}{\text { Mean }} \times 100
\end{aligned}
$$

\section{Acceptance criteria}

- Accuracy should be within $\pm 15 \%$ of the nominal concentrations except for LLOQ, where it should be within $\pm 20 \%$

- Precision determined at each concentration level should be $\pm 15 \%$ CV except, $\pm 20 \%$ CV at LLOQ

- Accuracy: Within-run and between runs: $\pm 15 \%$ of nominal concentrations; except $\pm 20 \%$ at LLOQ

- Precision: Within-run and between runs: $\pm 15 \%$ CV, except $\pm 20 \%$ CV at LLOQ

- Analytical runs which met the acceptance criteria will be reported and failed runs will be investigated

- The failed QCs can be removed from a calculation based on the outlier test. The data of runs with and without failed QC's shall be included in the report.

\section{Dilution integrity}

Dilution linearity demonstrates the accurate measurement of concentrations of spiked samples (i.e., QCs) exceeding the quantitation range when serially diluted to within the quantitative assay range. The A\&P of these diluted (DIQC) QC samples should be performed. Dilutions used during the validation shall mimic the expected dilutions in the study. Analyze a minimum of six replicates from DIQC samples against using the freshly prepared calibration standards along with freshly prepared QC samples [7,95-97].

\section{Acceptance criteria}

The mean accuracy of QC samples should be within $\pm 15 \%$ of the actual value after dilution and the precision determined at each dilution level should not exceed $15 \%$ of the $\mathrm{CV}$.

\section{Recovery}

Recovery refers to the extraction efficiency of an analytical method, reported as a percentage of the known amount of an analyte carried through the sample extraction and processing steps of the method.

\section{Absolute recovery}

Absolute recovery is calculated by relating the analyte/ISTD mean response found in extracted samples of low, medium, and high QC with analyte/ISTD mean response in neat aqueous solutions prepared at the low, medium, and high QC concentration.

$$
\begin{gathered}
\text { Average area (response) of } \\
\text { Absolute recovery }=\frac{\text { analyte peak in extracted sample }}{\text { Average area (response) of analyte }} \\
\text { in aqueoussample at } \\
\text { corresponding concentration }
\end{gathered}
$$

\section{Relative recovery}

Relative recovery is calculated by relating the analyte/ISTD mean response found in extracted samples of low, medium, and high QC with analyte/ISTD mean response in post-extraction spiked samples prepared at the low, medium, and high QC concentration.

$\begin{aligned} & \text { Average area (response) of analyte } \\ & \text { Relative recovery }= \frac{\text { peak in extracted sample }}{\text { Average area (response) of }} \\ & \text { analyte in postextractionspiked } \\ & \text { sample at corresponding concentration }\end{aligned}$

\section{Acceptance criteria}

The recovery for the analyte(s) is acceptable if $\% \mathrm{CV}$ is less than $15 \%$ for low, middle, and high QC concentration, individual recovery and global recovery should not be more than $110 \%[6,30]$.

\section{Matrix factor}

The biological matrix can play a role in affecting the selectivity, sensitivity and precision. This happens due to direct or indirect alteration of the response of analyte from the unintended interferences present in the biological matrix. Matrix factor dived into two types [65-70].

i. Absolute matrix factor

ii. Relative matrix factor.

Matrix factor determined by injecting Un-extracted low and high QC samples and the post-extraction spiked low and high QC samples.

Matrix effects can arise from several matrix components including, but not limited to:

- Endogenous biological components such as phospholipids, carbohydrates, and endogenous metabolites (bilirubin)

- Residual formulation components from intraperitoneal, intravenous, or oral dosing (PO) experiments; for example, polyethylene glycol, solutol, polysorbate (Tween 80 ), etc.

- An interaction between the analyte of interest and the matrix, such as covalent binding to plasma proteins or the enzymatic degradation of a prodrug

- Co-eluting drug metabolites, mobile phase additives, and concomitant medications.

Acceptance criteria

The $\%$ CV of the IS-normalized matrix factor calculated from the matrix lots should not be greater than 15\% [31-33].

\section{Stability}

Stability is a measure of the intactness of an analyte (lack of degradation) in a given matrix under specific storage and uses conditions relative to the starting material for given time intervals. Stability of the analyte in a given matrix should be determined, including the effects of sample collection, handling, and storage of the analyte [50-54]

Matrix-related stability experiments should compare stability QCs against freshly prepared calibration curves and freshly prepared QCs. Although the use of freshly prepared calibrators, QCs is the preferred approach [55-57],

Stability should assess in the same matrix as that intended for instudy samples; however, when the matrix is rare, the use of suitable surrogate matrices shall be utilized as per the respective study plan/ study requirement $[7,14,34]$.

The following stability shall be assessed during validation as per respective study plan [71].

a. Autosampler stability

b. Bench-top stability

c. Freeze-thaw stability

d. Wet extract stability

e. Dry extract stability

f. Reinjection reproducibility

g. Long-term stability of analyte in matrix

h. Stock/working solution stability

i. Whole blood stability.

Autosampler stability

The stability of the analyte and the internal standard should be assessed to cover the maximum period of anticipated subject sample batch run time. Place the processed samples in the auto sampler at the desired temperature and for the estimated period [72-76]. 


\section{Bench-top stability}

This is to establish the stability of a molecule for a period based on the expected duration that samples will be maintained at room temperature in the intended study. The spiked samples (LQC and HQC) it shall be kept at room temperature for at least for $6 \mathrm{~h}$ or more based on the duration of the intended study samples retained at room temperature [46-49].

\section{Freeze-thaw stability}

The stability of the analyte(s) in plasma samples during freeze-thaw cycles was determined by freezing the samples LQC and HQC in the freezer (at $-70^{\circ} \mathrm{C}$ and $-20^{\circ} \mathrm{C}$ ) and thawing them at room temperature. Analyte stability should be determined after a minimum of three freezing and thawing cycles [77-81].

\section{Wet extract stability}

Wet extract stability shall be evaluated at room temperature (approx $\left.25^{\circ} \mathrm{C}\right)$ and refrigerator $\left(2-8^{\circ} \mathrm{C}\right)$. Select the stability duration based on the characteristic of the Analyte(s) and to cover maximum anticipated sample processing time for the batch size to be used for subject sample analysis.

\section{Dry extract stability}

Dry extract stability should be performed only when the method includes evaporation step.

Process and store at least five replicates of LQC and HQC samples in a refrigerator $\left(2-8^{\circ} 0-70^{\circ} \mathrm{C}\right)$ without reconstitution after drying.

After the intended period, reconstitute and analyze the dry extract samples.

\section{Reinjection reproducibility}

Reproducibility is the precision between two laboratories. It also represents the precision of the method under the same operating conditions over a short period. Re-injection reproducibility shall be evaluated by re-injecting anyone of the accepted P \& A [82-85].

\section{Long-term stability of analyte in matrix}

Storage period in long-term stability should exceed the time between the first sample collection and the date of the last sample analysis (study samples). Long-term stability should be determined by the storage of at least six aliquots of each concentration (low, high, and diluted integrated QC) under the same conditions of the study samples [86,87].

\section{Stock and working solution stability}

The stability of stock and working solutions of analyte and the internal standard should be evaluated at room temperature for at least $6 \mathrm{~h}$. \% Deviation should be $\leq 10 \%$ of bias. If the stock and working solutions are refrigerated or frozen for the relevant period, the stability should be documented. After completion of the desired storage time interval, the stability should be tested by comparing the instrument response with that of freshly prepared stock and working solutions [58-61].

\section{Whole blood stability}

Whole blood stability is performed to ensure the stability of analyte in whole blood during sample collection, centrifugation, and handling, until plasma/serum has been separated. This period covers the duration taken for separation of plasma/serum during the conduct of the study [62-64].

Prepare test whole blood QC pools at LQC and HQC level. Store at room temperature/ice bath for 0 (control), 1 , and $2 \mathrm{~h}$. Centrifuge the whole blood sample and collect the plasma/serum (test matrix) for extraction and analysis following the validation method. Inject six replicates for each group. Compare the mean instrument response of stability test samples to that of the control group [3].

\section{Acceptance criteria}

The accuracy (\% nominal) at each level should be $\pm 1 \mathrm{~S} \%$ of the nominal value and precision should be $\pm 15 \%$ CV for all the above stabilities [7].

Hemolysis effect

Hemolysis effect is performed to ensure that the quantitation of the analyte is not affected in the presence of hemolysis of biological matrix (plasma and serum). Hemolysis effect was studied by processing six replicates of LQC and HQC concentrations spiked in hemolyzed plasma along with the calibration standards prepared in biological study matrix $[3,30]$.

Lipemic effect

The lipemic effect is performed to ensure that the quantitation of the analyte is not affected in the presence of the fed effect of a biological matrix (plasma, serum, and whole blood). The lipemic effect was studied by processing six replicates of LQC and HQC concentrations spiked in lipemic plasma along with the calibration standards prepared in biological study matrix $[3,30]$.

\section{Run size evaluation}

Evaluate the run size during method validation, which should include the number of samples to be analyzed under a run during actual study sample analysis. Establish run-size based on the chromatographic run time and analyte stability $[98,99]$

\section{Ruggedness (robustness)}

The robustness of an analytical procedure is a measure of its capacity to remain unaffected by small, but deliberate variations in method parameter condition such as small changes of $\mathrm{pH}$ values, mobile phase composition, and temperature and provide an indication of its reliability during typical usage. The assessment of robustness should be measured during the development phase and depends on the type of procedure under study. It should demonstrate the reliability of analysis concerning deliberate differences in method parameter conditions [14].

\section{CONCLUSION}

Bioanalysis turns into a primary tool to cover each stage of drug discovery to preclinical studies and all phases of clinical development. It provides not only selecting enhanced drug molecules but moreover improving our understanding of safety and pharmacokinetics of the drugs. A complete regulated bioanalytical method validation minimally obliges three precision and accuracy batch, stability and reproducibility experiments. Batch failures during method validation and study sample analysis are especially costly in terms of time, effort, and perception. The good practice to confirm the method validation is to conduct a comprehensive method development steps by way of increase the confidence of sponsor with the timely and successful completion of the study. GLP is an important issue in regulatory bioanalysis.

\section{AUTHORS' CONTRIBUTIONS OR CONFLICTS OF INTEREST}

All the authors have contributed equally. The author confirms that there are no conflicts of interest, financial, or otherwise.

\section{AUTHORS' FUNDING}

The author(s) received no financial support for the research, authorship, and/or publication of this article.

\section{REFERENCES}

1. Wenkui L, Jie Z, Francis LS. Handbook of LC-MS Bioanalysis. New Jersey: John Wiley \& Sons; 2013.

2. Shah VP, Midha KK, Findlay JW, Hill HM, Hulse JD, McGilveray IJ, et al. Bioanalytical method validation-A revisit with a decade of progress. Pharm Res 2000;17:1551-7.

3. Alan XU, Madden TL. LC-MS in Drug Bio-Analysis. Boston: Springer; 2012.

4. Venn RF. Principles and Practice of Bio-Analysis. London: Taylor \& Francis; 2000. 
5. Shah VP, Midha KK, Dighe S, McGilveray IJ, Skelly JP, Yacobi A, et al. Analytical methods validation: Bioavailability, bioequivalence and pharmacokinetic studies. Pharm Res 1992;9:588-92.

6. Kadian N, Raju KS, Rashid M, Malik MY, Taneja I, Wahajuddin M. Comparative assessment of bio-analytical method validation guidelines for pharmaceutical industry. J Pharm Biomed 2016;126:83-97.

7. F.D.A. U.S. Department of Health and Human Services, Center for Drug Evaluation and Research (CDER), Center of Veterinary Medicine (CVM) Guidance for Industry: Bio-Analytical Method Validation; 2018.

8. F.D.A. U.S. Department of Health and Human Services, Center for Drug Evaluation and Research (CDER), Center of Veterinary Medicine (CVM) Guidance for Industry: Bio-Analytical Method Validation; 2013.

9. F.D.A. U.S. Department of Health and Human Services, Center for Drug Evaluation and Research (CDER), Center of Veterinary Medicine (CVM) Guidance for Industry: Bio-Analytical Method Validation; 2001.

10. ANVISA, Agencia Nacional de Vigilancia Sanitaria, Guide for Validation of Analytical and Bio-Analytical Methods Resolution-RE No. 899; 2003.

11. ANVISA, Ministry of Health, National Health Surveillance Agency, Resolution-RDC No. 27; 2012

12. EMA, Committee for Medicinal Product for Human Use Guideline on Bio-Analytical Method Validation; 2011.

13. MHLW, Guideline on Bio-Analytical Method Validation in Pharmaceutical Development; 2013.

14. ICH. International Council for Harmonisation of Technical Requirements for Pharmaceuticals for Human Use: Bio-Analytical Method Validation M10; 2019.

15. Niazi SK. Handbook of Bioequivalence Testing. New York: Informa Healthcare; 2007.

16. Rifai N, Horvath AR, Wittwer CT, Hoofnagle A, editors. Principles and Applications of Clinical Mass Spectrometry. $5^{\text {th }}$ ed. Netherlands: Elsevier; 2018. p. 67-91.

17. Snyder LR, Kirkland JJ, Glajch JL. Practical HPLC Method Development. $2^{\text {nd }}$ ed. United States: John Wiley \& Sons; 2001.

18. Tijare LK, Rangari NT, Mahajan UN. A review on bioanalytical method development and validation. Asian J Pharm Clin Re 2016;9:6-10.

19. Sargent M. Guide to Achieving Reliable Quantitative LC-MS Measurements, RSC Analytical Methods Committee; 2013.

20. Tiwari G, Tiwari R. Bioanalytical method validation: An updated review. Pharm Methods 2010;1:25-38.

21. Mano Y. Method validation studies and an inter-laboratory cross validation study of lenvatinib assay in human plasma using LC-MS MS. Pract Lab Med 2018;12:e0103.

22. Viswanathan CT, Bansal S, Booth B, DeStefano AJ, Rose MJ, Sailstad J, et al. Quantitative bioanalytical methods validation and implementation: Best practices for chromatographic and ligand binding assays. AAPS J 2007;9:E260-7.

23. Satheshkumar S, Muruganantham V. A simple and rugged bio-analytical method development and validation of brivudine in human plasma by using high-performance liquid chromatography. Int J Pharm Pharm Sci 2010;12:45-50.

24. Dadgar D, Burnett PE. Issues in evaluation of bioanalytical method selectivity and drug stability. J Pharm Biomed 1995;14:23-31.

25. Ingelse B, Barroso B, Gray N, Jakob-Rodamer V, Kingsley C, Sykora C, et al. European bioanalysis forum: Recommendation on dealing with hemolyzed and hyperlipidemic matrices. Bioanalysis 2014;6:3113-20.

26. Scientific Working Group for Forensic Toxicology. Scientific working group for forensic toxicology (SWGTOX) standard practices for method validation in forensic toxicology. J Anal Toxicol 2013;37:452-74.

27. International Conference on Harmonization (ICH). Validation of Analytical Methods: Methodology, International Conference on Harmonization Q2 B; 1996.

28. Thompson M, Ellison SL, Wood R. Harmonized guidelines for single laboratory validation of method of analysis. Pure Appl Chem 2008;74:835-55.

29. Saudagar RB, Thete PG. Bioanalytical method validation: A concise review. Asian J Pharm Sci 2018;8:107-14.

30. Kollipara S, Bende G, Agarwal N, Varshney B, Paliwal J. International guidelines for bioanalytical method validation: A comparison and discussion on current scenario. Chromatographia 2011;73:201-17.

31. Hall TG, Smukste I, Bresciano KR, Wang Y, McKearn D, Savage RE. Identifying and overcoming matrix effects in drug discovery and development. In: Prasain JK, editor. Tandem Mass SpectrometryApplications and Principles. London: Intech Open; 2012. p. 389-421.
32. Braggio S, Barnaby RJ, Grosi P, Cugola. A strategy for validation of bioanalytical methods. J Pharm Biomed 1996;14:375-88.

33. Bansal S, DeStefano A. Key elements of bio-analytical method validation for small molecules. AAPS J 2007;9:109-14.

34. Boer T, Wieling J. Incurred sample accuracy assessment: Design of experiments based on standard addition. Bioanalysis 2011;3:983-92.

35. Wood R. How to validate analytical methods. Trends Analyt Chem 2005;18:624-32.

36. McDowall RD. The role of laboratory information management systems LIMS in analytical method validation. Anal Chim Acta 2007;54:149-58.

37. Heyden YV, Nijhuis A, Smeyers-Verbeke J, Vandeginste BG, Massart DL. Guidance for robustness/ruggedness tests in method validation. J Pharm Biomed Anal 2001;24:723-53.

38. Puluido A, Ruusanches I, Boque R, Rius FX. Uncertainty of results in routine qualitative analysis in analytical chemistry. J Pharm Biomed Anal 2005;22:647-54.

39. Jhang JS, Chang CC, Fink DJ, Kroll MH. Evaluation of linearity in the clinical laboratory. Arch Pathol Lab Med 2004;128:44-8.

40. Mark H. Application of an improved procedure for testing the linearity of analytical methods to pharmaceutical analysis. J Pharm Biomed Anal 2003;33:7-20.

41. Trullols E, Ruisanchez I, Rius FX. Trends in analytical chemistry. J Lab Invest 2003;23:137-45

42. Valcarcel M, Cardenas S, Gallego M. Sample screening system in analytical chemistry. Trends Analyt Chem 1999;18:685-94.

43. Ye C, Liu J, Ren F, Okafo N. Design of experiment and data analysis by JMP (SAS institute) in analytical method validation. J Pharm Biomed Anal 2000;23:581-9.

44. Lindner W, Wainer IW. Requirements for initial assay validation and publication in J. Chromatography B. J Chromatogr B Biomed Sci Appl 1998;707:1-2.

45. Penninckx W, Hartmann C, Massart DL, Smeyers-Verbeke J. Validation of the calibration procedure in atomic absorption spectrometric methods. J Anal At Spectrom 1998;11:237-46.

46. Nowatzke W, Woolf E. Best practices during bio-analytical method validation for the characterization of assay reagents and the evaluation of analyte stability in assay standards, quality controls, and study samples. AAPS J 2007;9:E117-22.

47. James CA, Breda M, Frigerio E. Bio-analytical method validation: A risk-based approach? J Pharm Biomed Anal 2004;35:887-93.

48. Nakashima K. High-performance liquid chromatography of drug of abuse in biological samples. J Health Sci 2009;51:272-7.

49. Boulanger B, Chiap P, Dewe W, Crommen J, Hubert P. An analysis of the SFSTP guide on validation of chromatographic bioanalytical methods: Progress and limitations. J Pharm Biomed Anal 2003;32:753-65.

50. Causon R. Validation of chromatographic methods in biomedical analysis. Viewpoint and discussion. J Chromatogr B Biomed Sci Appl 1997;689:175-80

51. Hartmann C, Smeyers-Verbeke J, Massart DL, McDowall RD. Validation of bioanalytical chromatographic methods. J Pharm Biomed Anal 1998; 17:193-218

52. Hubert P, Chiap P, Crommena J, Boulanger B, Chapuzet EN, Laurentie $\mathrm{M}$, et al. The SFSTP guide on the validation of chromatographic methods for drug bio-analysis: From the Washington Conference to the laboratory. Anal Chim Acta 1999;391:135-48

53. Zhoua S, Songb Q, Tangb Y, Weng N. Critical review of development, validation, and transfer for high throughput bio-analytical LC-MS/MS methods. Curr Pharm Anal 2005;55:3-14.

54. Tabrizi-Fard MA, Fung HL. Reversed-phase high-performance liquid chromatography method for the analysis of nitro-arginine in rat plasma and urine. J Chromatogr B Biomed Appl 1996;679:7-12.

55. Hartmann C, Massart DL, McDowall RD. An analysis of the Washington Conference report on bio-analytical method validation. J Pharm Biomed Anal 1994;12:1337-43.

56. Wieling J, Hendriks G, Tamminga WJ, Hempenius J, Mensink CK, Oosterhuis B, et al. Rational experimental design for bio-analytical methods validation. Illustration using an assay method for total captopril in plasma. J Chromatogr 2006;730:381-94.

57. Miller KJ, Bowsher RR, Celniker A, Gibbons J, Gupta S, Lee JW, et al. Workshop on bioanalytical methods validation for macromolecules: Summary report. Pharm Res 2001;18:1373-83.

58. Kringle R, Hoffman D. Stability methods for assessing stability of compounds in whole blood for clinical bioanalysis. Drug Inf $\mathrm{J}$ 2001;35:1261-70.

59. Chiu ML, Lawi W, Snyder ST, Wong PK, Liao JC, Gau V. Matrix effects: A challenge toward automation of molecular analysis. J Assoc Lab Autom 2010;15:233-42. 
60. Reid E, Wilson ID. Methodological survey in biochemistry and analysis. In: Analysis for Drug and Metabolites, Including Anti-Infective Agents. Vol. 20. Cambridge, England: Royal Society of Chemistry; 1990. p. 1-57.

61. Kallner A. Quality specifications based on the uncertainty of measurement. Scand J Clin Lab Invest 1999;59:513-6.

62. Rao KR, Kumar KS. Bioanalytical method validation-a quality assurance auditor view point. J Pharm Sci Res 2009;1:1-10.

63. Lang JR, Bolton S. A comprehensive method validation strategy for bio-analytical applications in the pharmaceutical industry. Experimental considerations. J Pharm Biomed Anal 1991;9:357-61.

64. Shah VP. The history of bio-analytical method validation and regulation: Evolution of a guidance document on bio-analytical method validation. AAPS J 2007;9:43-7.

65. Buick AR, Doig MV, Jeal SC, Land GS, McDowall RD. Method validation in the bio-analytical laboratory. J Pharm Biomed Anal 1990;8:629-37.

66. Mark H. Application of improved procedure for testing linearity of analytical method to pharmaceutical analysis. J Pharm Biomed Anal 2003;33:7-20.

67. Wells DA. High throughput bio-analytical sample preparation: Methods and automation strategies. In: Progress in Pharmaceutical and Biomedical Analysis. Vol. 30. Amsterdam, London: Elsevier; 2003. p. 610.

68. Karnes HT, Shiu G, Shah VP. Validation of bio-analytical methods. Pharm Res 1991;8:421-6.

69. Kringle RO. An assessment of the 4-6-20 rule of acceptance of analytical runs in bioavailability, bioequivalence, and pharmacokinetic studies. Pharm Res 1994;11:556-60.

70. Singh PS, Shah G. Analytical method development and validation. J Pharm Res 2011;4:2330-2.

71. Timm U, Wall M, Dell D. A new approach for dealing with the stability of drugs in biological fluids. J Pharm Sci 1985;74:972-7.

72. Buszewski B, Szultka M. Past, Present, and Future of Solid Phase Extraction: A Review, Crit. Rev. Anal. Chem., 2012;42:198-13.

73. Satheshkumar S, Muruganatham V. Development of validated bioanalytical RP-HPLC method for determination of sofosbuvir in human plasma. Am J Pharm Tech Res 2020;10:82-92.

74. Madhavi S, Rani AP. Bio-analytical method development and validation for the determination of sofosbuvir from human plasma. Int J Pharm Pharm Sci 2017;9:35-41.

75. Vikas PM, Satyanarayana T, Kumar DV, Mounika E, Latha MS, Anusha R, et al. Development and validation of new RP-HPLC method for the determination of sofosbuvir in pure form. World J Pharm Pharm Sci 2016;5:775-81

76. Mamdouh R, Emad RB, Karim BI. Development of a sensitive UPLCESI-MS/MS method for quantification of sofosbuvir and its metabolite, GS-331007 in human plasma: Application to a bioequivalence study. J Pharm Biomed Anal 2015;114:97-104

77. Gandhi BM, Rao AL, Rao JV. UPLC-MS/MS method for determination of sofosbuvir in human plasma. Ann Pharm Fr 2017;75:257-66.

78. Yang H, Robinson M, Corsa AC, Peng B, Cheng G, Tian Y, et al. Preclinical characterization of the novel hepatitis C Virus NS3 protease inhibitor GS-9451. Antimicrob Agents Chemother 2014;58:647-53.

79. Ayisi NK. High-performance liquid chromatographlc analysis of (E)5-(2-bromovinyl)-Z'deoxyuridine and its metabolites in serum, urine and herpes simplex virus Type-1 infected cells. J Chromatogr 1986;375: 423-30.

80. Reeuwijk HJ, Lingeman H, Tjaden UR, de Bruijn EA, Keizer HJ, van der Greef J. Bioanalysis of (E)-5-(2-bromovinyl)-2'-deoxyuridine. J Chromatogr 1988;428:93-102.

81. Peng XD, Tan ZR, Wu HZ, Zhou G, Guo CX, Pei Q, et al. LCMS-MS quantitative determination of brivudine in human plasma and its application to pharmacokinetic studies. Chromatographia 2011;73:1089-95.

82. Dyade GK, Sawant RL. Simple bioanalytical quantification method for simultaneous estimation of simvastatin and ezetimibe in human plasma by reverse-phase high-performance liquid chromatography technique. Asian J Pharm Clin Res 2020;13:160-5.

83. Yin P, Lehmann R, Xu G. Effects of pre-analytical processes on blood samples used in metabolomics studies. Anal Bioanal Chem 2015;407:4879-92.

84. Deglon J, Leuthold LA, Thomas A. Potential missing steps for a wide use of dried matrix spots in biomedical analysis. Bioanalysis 2015;7:2375-85.

85. Li W. Evaluation of plasma microsampling for dried plasma spots (DPS) in quantitative LC-MS/MS bio-analysis using ritonavir as a model compound. J Chromatogr B Analyt Technol Biomed Life Sci 2015;991:46-52

86. Sen A. Metabolic phenotype of the healthy rodent model using in-vial extraction of dried serum, urine, and cerebrospinal fluid spots. Anal Chem 2013;85:7257-63.

87. Dong Y. A sensitive dilute-and-shoot approach for the simultaneous screening of 71 stimulants and 7 metabolites in human urine by LCMS-MS with dynamic MRM. J Chromatogr Sci 2015;53:1528-35.

88. Cao Z, Kaleta E, Wang P. Simultaneous quantitation of 78 Drugs and metabolites in urine with a dilute-and-shoot LC-MS-MS assay. J Anal Toxicol 2015;39:335-46.

89. Rodin I. 'Dilute-and-shoot' RSLC-MS-MS method for fast detection of nerve and vesicant chemical warfare agent metabolites in urine. J Anal Toxicol 2015;39:69-74

90. Kohler I, Schappler J, Rudaz J, Microextraction techniques combined with capillary electrophoresis in bio-analysis. Anal Bioanal Chem 2013;405:125-41.

91. Silva C. Microextraction by packed sorbent (MEPS) and solid-phase microextraction (SPME) as sample preparation procedures for the metabolomic profiling of urine. Metabolites 2014;4:71-97.

92. Bojko B. Low invasive in vivo tissue sampling for monitoring biomarkers and drugs during surgery. Lab Invest 2014;94:586-94.

93. Cudjoe E. Solid-phase microextraction: A complementary in vivo sampling method to microdialysis. Angew Chem Int Ed Engl 2013;52:12124-6.

94. Thulasiraman, V. Reduction of the concentration difference of proteins in biological liquids using a library of combinatorial ligands. Electrophoresis 2005;26:3561-71.

95. Staub A. Analysis of hemoglobin-based oxygen carriers by CE-UV/Vis and CE-ESITOF/MS. Electrophoresis 2010;31:1241-7.

96. Rezaee M. Determination of organic compounds in water using dispersive liquid-liquid microextraction. J Chromatogr A 2006;1116:1-9.

97. Zuloaga O. Dispersive liquid-liquid microextraction: Trends in the analysis of biological samples. Bioanalysis 2015;7:2211-25.

98. Li Y. Development of a efficient and sensitive dispersive liquid-liquid microextraction technique for extraction and preconcentration of 10 beta2-agonists in animal urine. PLoS One 2015;10:e0137194.

99. Kohler I. Dispersive liquid-liquid microextraction combined with capillary electrophoresis and time-of-flight mass spectrometry for urine analysis. J Pharm Biomed Anal 2013;73:82-9. 